\title{
Endothelial cell loss rate after penetrating keratoplasty: Optical versus therapeutic grafts
}

\author{
Abdelrhman Shams ${ }^{1}$, Ayman Abdelmoneim Gaafar ${ }^{1}$, Rania Serag Elkitkat ${ }^{1,2,3}$ and Mohamed Omar Yousif ${ }^{1,4}$ \\ ${ }^{1}$ Department of Ophthalmology, Faculty of Medicine, Ain Shams University, Cairo, Egypt \\ ${ }^{2}$ Watany Eye Hospitals, Cairo, Egypt \\ ${ }^{3}$ Watany Research and Development Center, Cairo, Egypt \\ ${ }^{4}$ Cornea and Refractive Surgery Consultant, Maadi Eye Subspecialty Center, Cairo, Egypt
}

\begin{abstract}
Background: This study aimed to compare the rate of endothelial cell loss (ECL) after penetrating keratoplasty (PKP) for optical versus therapeutic grafts at 3-, 6-, and 12-month postoperatively. Furthermore, the study aimed to investigate postoperative graft viability and the rate of graft rejection during the first year of follow-up for both indications.

Methods: This was a prospective, observational, comparative study that included patients who sought medical advice at the cornea outpatient clinic of Ain Shams University Hospitals, Cairo, Egypt. The study recruited 60 patients: group 1 included 30 transplanted corneas of 30 patients who underwent optical PKP for various indications, while group 2 included 30 transplanted corneas of 30 patients who underwent therapeutic PKP for unhealed, resistant infectious keratitis. Specular microscopy was performed for all patients at the 3-, 6-, and 12-month follow-up visits using Nidek CEM-530 specular microscopy. Postoperative clinical examinations were performed at the same follow-up visits to detect graft rejection.

Results: There were no statistically significant differences between the groups concerning the postoperative timing of graft clarity or the rate of ECL at 3- and 6-months postoperatively; however, the rate of ECL was significantly greater in group 2 than in group 1 at 12 -months postoperatively $(P=0.03)$, although the difference was small from a clinical point of view. Moreover, there was no statistically significant difference between the groups in terms of the graft rejection rate.

Conclusions: Therapeutic PKP results were comparable to optical PKP with respect to graft viability, the rate of ECL, and the rate of graft rejection 1 year after grafting.

\section{KEY WORDS}

penetrating keratoplasty, PKP, optical, therapeutic, endothelial cell density, endothelial cell loss, specular microscopy, graft rejection
\end{abstract}

\section{INTRODUCTION}

Corneal diseases are the second major cause of blindness after cataracts, worldwide. They comprise a wide range of ectatic, infectious, and inflammatory disorders. The prevalence of corneal diseases varies across different countries and populations, depending on the availability and general standards of eye care [1].

The mainstay of treatment for infectious keratitis is frequent application of topical antimicrobial agents. Although this treatment modality commonly eliminates the infectious agent, there is an increasing incidence

Correspondence: Abdelrhman Shams, MD, Assistant Lecturer of Ophthalmology, Faculty of Medicine, Ain Shams University, Cairo, Egypt. Email: abdelrhmanshams@hotmail.com. ORCID iD: https://orcid.org/0000-0001-7313-549X

How to cite this article: Shams A, Abdelmoneim Gaafar A, Elkitkat RS, Omar Yousif M. Endothelial cell loss rate after penetrating keratoplasty: Optical versus therapeutic grafts. Med Hypothesis Discov Innov Ophthalmol. 2021 Summer; 10(2): 74-79. https://doi.org/10.51329/mehdiophthal1424

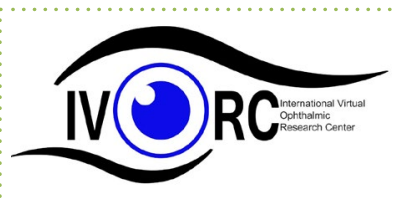

Copyright (C) Author(s). This is an open-access article distributed under the terms of the Creative Commons Attribution-NonCommercial 4.0 International License (http://creativecommons.org/licenses/by-nc/4.0/) which permits copy and redistribute the material just in noncommercial usages, provided the original work is properly cited. (c) (i) (s) 
of antimicrobial resistance [2, 3]. Accordingly, other modalities for proper management should be used, such as keratoplasty, which may be an effective surgical intervention. In addition to resistance to antimicrobial agents, other indications for surgical intervention in corneal infections include progressive corneal thinning, descemetocele, and perforated corneal ulcers [4]. Thus, therapeutic keratoplasty may be needed in a significant proportion of corneas with antimicrobial resistance or complicated infectious keratitis. In addition to therapeutic keratoplasty, optical keratoplasty is another common indication for corneal transplantation in corneas with significant scars after keratoconus $(\mathrm{KCN})$ or healed infectious keratitis. It can help to restore corneal clarity and thus attain good visual performance [5].

After penetrating keratoplasty (PKP), either for therapeutic or optical indications, both postoperative visual acuity and graft clarity are related to many complex physiological and immunological conditions, as restoring the anatomical integrity of the globe cannot guarantee vision improvement [6]. Previous studies have demonstrated the importance of both endothelial cell density (ECD) and rate of endothelial cell loss (ECL) as major physiological determinants for maintaining graft clarity and survival after PKP $[7,8]$.

The ECD of the corneal donor is an unstable parameter that is amenable to change with time. In a transplanted corneal graft, progressive ECL can occur in many instances. The underlying reasons for this observed ECL, even years after PKP, are poorly understood. Some corneal donor factors have been shown to play a major role; these include donor age, corneal donor ECD, death-to-preservation time, and type of storage medium. Additionally, recipient factors have also been reported to affect the ECL rate, such as significant surgical trauma, the development of intraocular pressure (IOP) spikes, and/or graft rejection [9].

To the best of our knowledge, few studies have compared the ECL rate after optical versus therapeutic PKP $[10,11]$. Hence, the present study compared the ECL rate for optical versus therapeutic grafts at 3,6 , and 12 months after grafting. Furthermore, this study aimed to investigate the postoperative graft viability and rate of graft rejection during the first year of follow-up for both indications.

\section{METHODS}

This was a prospective, observational, comparative study that included patients who sought medical advice at the Cornea Outpatient Clinic of Ain Shams University Hospitals, Cairo, Egypt. All enrolled subjects attended the clinic in the period from July 2019 to June 2020. The study adhered to the tenets of the Declaration of Helsinki and was approved by the Ethical Committee of Ain Shams University (Ethical code: FWA000017585). All participants were informed about the nature of the study, and signed informed consent prior to participation.

The study included patients aged 17-76 years who underwent PKP surgery. We excluded candidates with any clinically detectable posterior segment pathology and any corneas with factors that could possibly alter the postoperative ECL rate, including preoperative glaucoma, the occurrence of any intraoperative complications that would compromise the corneal endothelium, any postoperative increase in IOP, steroid responders, and any detectable postoperative attacks of endothelial graft rejection. The study recruited 60 patients: group 1 included 30 transplanted corneas of 30 patients who underwent optical PKP for various indications, while group 2 included 30 transplanted corneas of 30 patients who underwent therapeutic PKP for unhealed, resistant infectious keratitis.

For all participants, a full ophthalmological examination was performed before and after the surgical intervention, including uncorrected distance visual acuity (UDVA), corrected distance visual acuity (CDVA) (both performed using a Snellen visual acuity chart, and then converted to logarithm of the minimum angle of resolution [logMAR] visual acuity), slit lamp examination (SL-D701 slit lamp, Topcon, Tokyo, Japan), and fundus examination using fundus biomicroscopy (whenever possible). Other investigations were also performed when required. Specifically, B-scan sonography (Echoscan US- 4000, NIDEK Co. Ltd., Bunkyo, Japan) was performed in cases with blurred or no fundus view (4 patients in group 1 and 5 patients in group 2). Moreover, electrophysiological tests were performed to determine the postoperative visual potential using flash electroretinography and flash visual evoked potential (Reti-port, Roland Consult, Electrophysiological Diagnostic System, Brandenburg, Germany) in cases with poor vision and significant corneal opacification (3 patients in group 1 and 5 patients in group 2).

For all enrolled patients in the study, PKP was performed using corneal grafts with a viability of no more than the $11^{\text {th }}$ day post-preservation, where all corneal grafts were preserved in Optisol-GS (OGS, Bausch \& Lomb Surgical, Irvine, CA, USA). In addition, all the enrolled donor grafts were sutured to the recipients' ocular beds using a fixed number of sutures (16 interrupted 10-0 monofilament nylon sutures in all cases). The punch and trephine sizes were selected according to the recipient's corneal size or peripheral extent of corneal infection. All enrolled patients underwent PKP with no cataract extraction, and all included PKPs were performed by the same experienced surgeon (M.O.Y.). 
Postoperatively, all recruited patients attended their follow-up examinations (scheduled on the $3^{\text {rd }}$ day, $10^{\text {th }}$ day, and then at 1-, 3-, 6-, and 12-months postoperatively). Standard postoperative treatment was prescribed for patients in both groups. This included prednisolone acetate ophthalmic suspension 1\% (Pred Forte; Allergan, Inc., Irvine, CA, USA), which was administered 6 times daily for 10 days, then tapered off along a 1-year interval, reducing by 1 drop application every 3 months. This was combined with topical moxifloxacin (Vigamox, Alcon, Novartis Pharma AG, Basel, Switzerland) 3 times daily for 10 days or until full epithelization of the graft. Regarding the therapeutic PKP group, 12 cases were categorized as cases at high risk of re-infection, based on the preoperative peripheral location of the infection. These patients received intense antimicrobial treatment postoperatively (every 2 hours for 2-3 weeks until no re-infection occurred in the graft bed). The antimicrobials were fortified topical antibiotics, including fortified vancomycin $(25 \mathrm{mg} / \mathrm{ml}$ vancomycin; ANI Pharmaceuticals, Inc., Baudette, MN, USA), and fortified ceftazidime ( $50 \mathrm{mg} / \mathrm{ml}, 1-\mathrm{g}$ vial, Fortum, GlaxoSmithKline, Brentford, UK). For fungal infections, topical fluconazole ( $2 \mathrm{mg} / \mathrm{ml}$ Diflucan, Pfizer, New York, NY, USA) was added following the same regimen of anti-microbials.

In the enrolled participants, the grafts were considered clear postoperatively when there were no detected Descemet's or stromal folds on slit lamp examination, as compared with the other normal eye of the patient Specular microscopy was performed for all participants at the 3-, 6-, and 12-months postoperative visits, using a Nidek CEM-530 specular microscope (Nidek Co, Ltd, Gamagori, Japan). The images were captured according to the user instructions of the device [12]. The subjects were seated and positioned appropriately. Then, they were asked to fixate on the internal target within the device, followed by a rapid complete blink to ensure a smooth spread of the tear film. The corneal endothelium was evaluated in the central area using auto-analysis. ECD was recorded and analyzed.

Data were collected, revised, coded, and analyzed using the Statistical Package for Social Science software for Windows (version 23.0; IBM Corp., Armonk, NY, USA). Quantitative data are presented as mean and standard deviation (SD), and range (parametric), while qualitative variables were presented as numbers and percentages. Comparisons between groups were performed using the chi-square test for qualitative data and independent samples $t$-test for quantitative data. The confidence interval (CI) was set at $95 \%$, and the accepted margin of error was set at $5 \%$. Statistical significance was set at $P<0.05$.

\section{RESULTS}

The study recruited 60 eyes of 60 patients (30 eyes in each group); however, 5 eyes were excluded from group 1 ( 2 eyes with endothelial graft rejection episodes and another 3 eyes due to raised IOP postoperatively), and 10 eyes were excluded from group 2 ( 6 eyes with endothelial graft rejection episodes, 2 eyes due to raised IOP postoperatively, and 2 eyes due to graft re-infection). Therefore, the final statistical analysis was performed on 25 patients in group 1 and 20 patients in group 2. The mean \pm SD of age in groups 1 and 2 was $43.70 \pm 14.79$, and $47.27 \pm 14.51$ years, respectively $(P=0.350)$. The female/male ratio was $1: 1.33$ for group 1 and $1: 1.42$ for group $2(P=0.426)$, and the percentage of right to left eyes was $46.7 \%$ to $53.3 \%$ for group $1(P=0.325)$ and $60 \%$ to $40 \%$ for group $2(P=0.301)$. There were no statistically significant differences between the 2 groups in any of the demographic parameters. Moreover, the preoperative IOP measurements, fundus biomicroscopy, and B-scan sonography or electrophysiological tests were all unremarkable. No significant lens opacity was detected in any of the enrolled participants.

The indications for PKP, the donor's age, the time-interval from death to graft preservation, the preservation time of the graft, and the initial ECD of the donor's graft for both groups are detailed in Table 1. There were no statistically significant differences between the groups in any of the aforementioned parameters, except for the ECD of the graft, which was significantly lower in group 2 than in group $1(P<0.01)$.

With regard to graft clarity, $90.4 \%$ and $77.2 \%$ of the grafts in groups 1 and 2, respectively, gained clarity on the $10^{\text {th }}$ postoperative day, with no statistically significant difference between the groups. The graft clarity had reached $100 \%$ for both groups at the 1-month follow-up visit.

On performing slit lamp assessment of the anterior segment postoperatively, when the corneal clarity permitted a proper evaluation of the crystalline lens, no cataracts were detected in any of the examined eyes.

The ECL rate (Table 2$)$ showed no statistically significant differences between groups at $3(P=0.141)$ and 6-months $(P=0.972)$ postoperatively, yet the rate of ECL was significantly greater in group 2 than in group 1 at 12 -months postoperatively $(P<0.05)$. Two grafted corneas in groups 1 and 6 grafted corneas in group 2 developed endothelial graft rejection episodes, but the difference between the 2 groups was statistically insignificant $(P=0.129)$. There was also no statistically significant difference in terms of the number of steroid responders between the groups ( 3 eyes in group 1 and 2 eyes in group $2, P=0.640)$. In addition, only 2 grafted corneas in group 2 developed fungal re-infection. 
Table 1. Demographic and preoperative characteristics of the studied participants and details of the grafted corneas in the 2 groups

\begin{tabular}{|c|c|c|c|c|}
\hline \multicolumn{2}{|l|}{ Variable } & \multirow{2}{*}{$\begin{array}{l}\text { Group } 1(\mathbf{n}=\mathbf{3 0}) \\
13(43.3)\end{array}$} & \multirow{2}{*}{$\begin{array}{l}\text { Group } 2(\mathbf{n}=\mathbf{3 0}) \\
10(33.3)\end{array}$} & \multirow{3}{*}{$\begin{array}{l}\text { P-value } \\
0.426^{*}\end{array}$} \\
\hline \multirow{2}{*}{ Recipient sex, n (\%) } & Female & & & \\
\hline & Male & $17(56.7)$ & $20(66.7)$ & \\
\hline Recipient age (years) & Mean \pm SD (Range) & $43.70 \pm 14.79(19$ to 72$)$ & $47.27 \pm 14.51(17$ to 76$)$ & $0.350^{* *}$ \\
\hline \multirow{2}{*}{ Operated eye, n (\%) } & Right & $14(46.7$ & $18(60.0)$ & \multirow{2}{*}{$0.301^{* *}$} \\
\hline & Left & $16(53.3)$ & $12(40.0)$ & \\
\hline \multirow{6}{*}{ Recipient diagnoses, n (\%) } & Fungal keratitis & - & $19(63.3)$ & - \\
\hline & Bacterial keratitis & - & $11(36.7)$ & - \\
\hline & Old corneal opacity & $11(36.7)$ & - & - \\
\hline & Traumatic scar & $12(40.0)$ & - & - \\
\hline & Decompensation & $4(13.3)$ & - & - \\
\hline & Keratoconus & $3(10.0)$ & - & - \\
\hline Corneal donor ECD & Mean \pm SD (Range) & $2528.47 \pm 348.32(1900$ to 3143$)$ & $2196.43 \pm 236.86(1800$ to 2750$)$ & $<0.01^{* *}$ \\
\hline Donor age (years) & Mean \pm SD (Range) & $48.53 \pm 13.37(4$ to 66$)$ & $54.60 \pm 12.44(25$ to 82$)$ & $0.074^{* *}$ \\
\hline Donor death to PT (hours) & Mean \pm SD (Range) & $7.97 \pm 3.78(3$ to 18$)$ & $8.37 \pm 3.93(3$ to 15$)$ & $0.689^{* *}$ \\
\hline Donor PT (days) & Mean \pm SD (Range) & $9.23 \pm 1.10(8$ to 11$)$ & $9.27 \pm 1.39(7$ to 11$)$ & $0.918^{* *}$ \\
\hline
\end{tabular}

Abbreviations: n, number; PKP, penetrating keratoplasty; SD, standard deviation; ECD, endothelial cell density; PT, preservation time. $P$-value $<0.05$ is shown in bold $\left({ }^{*} \mathrm{Chi}\right.$-square test; ${ }^{* *}$ : Independent samples $\mathrm{t}$-test $)$. Note: Decompensation, decompensation due to pseudophakic bullous keratopathy with scarred corneal stroma; Group 1, optical PKP; Group 2, therapeutic PKP.

Table 2. The difference in the rate of endothelial cell loss between Group 1 (optical PKP) and Group 2 (therapeutic PKP) after excluding eyes with endothelial graft rejection episodes

\begin{tabular}{|l|l|l|l|}
\hline Examination time point & $\begin{array}{l}\text { Group } 1 \text { (n= 25) } \\
\text { Mean } \pm \text { SD (Range) }\end{array}$ & $\begin{array}{l}\text { Group 2 (n= 20) } \\
\text { Mean } \pm \text { SD (Range) }\end{array}$ & \multicolumn{1}{|l|}{ P-value } \\
\hline 3-months postop & $29.90 \pm 2.19(27.5$ to 34.04$)$ & $30.77 \pm 1.53(28$ to 32.43) & 0.141 \\
\hline 6-months postop & $39.27 \pm 1.97(37.5$ to 42.03$)$ & $39.29 \pm 1.91(37$ to 42.03$)$ & 0.972 \\
\hline 12-months postop & $49.06 \pm 2.83(46$ to 53.02$)$ & $50.65 \pm 1.59(48$ to 53.85$)$ & $\mathbf{0 . 0 3 0}$ \\
\hline
\end{tabular}

Abbreviations: PKP, penetrating keratoplasty; postop, postoperatively; $\mathrm{n}$, number; $\mathrm{SD}$, standard deviation; $\mathrm{m}$, months. $P$-value $<0.05$ is shown in bold (independent $t$-test).

\section{DISCUSSION}

The present study evaluated graft survival and compared the rate of ECL during the first postoperative year following both optical and therapeutic PKPs. The corneal grafts that were included in the ECL analysis in both groups showed $100 \%$ graft clarity in all cases after 1 month, and remained clear until the end of the study period. The ECL rate for both groups was not significantly different, except at the 12-months visit, where there was a statistically significant (yet clinically small) increase in the rate of ECL in group 2 as compared to group 1 . Furthermore, the present study showed no statistically significant differences between the groups with regard to the rate of endothelial graft rejection. Hence, given the comparable results between the groups in terms of regaining graft clarity, the ECL rate, and the graft rejection rate, our study results may indicate that therapeutic PKP could be comparable to optical PKP in terms of graft viability and postoperative prognosis during the first year of follow-up.

In our study, there was a significant difference between the groups in terms of the initial ECD of the donor's graft, where patients in group 1 (optical PKP) had significantly higher ECD values than those in group 2 (therapeutic PKP). This can be explained by the usual tendency toward selecting better grafts for cases requiring optical PKP than for those requiring therapeutic PKPs, since the former are claimed to have a better prognosis [9]. In addition, the emergency nature of therapeutic PKPs sometimes requires surgeons to use available grafts [13]. Nevertheless, we believe that this did not alter the credibility of our study results, as we mainly compared the 2 groups in terms of the ECL rate, rather than the ECD alone.

In the present study, the ECL rate in group 1 was $29.90 \% \pm 2.19,39.27 \% \pm 1.97$, and $49.06 \% \pm 2.83 \%$ at the 3-, 6-, and 12-month intervals, respectively, while it was $30.77 \% \pm 1.53 \%, 39.29 \% \pm 1.91 \%$, and $50.65 \% \pm 1.59 \%$, respectively, in group 2. For the optical PKP cases, the rate of ECL was comparable to that reported by Obata et 
al. [14], where the postoperative ECL rate was $10.4 \%$ at 2 weeks, $16.3 \%$ at 1 month, $33.6 \%$ at 3 months, $39.4 \%$ at 6 months, and $48.2 \%$ at 12 months. This was similar to the findings of other studies by Bourne et al. [15], Nishimura et al. [16], and Patel et al. [7], in which the greatest ECL rate after optical PKP occurred during the first postoperative year, with a collective rate of 30-50\%, which was comparable to our study results. Another study by Eghtedari et al. [17], which was performed on cases with optical PKPs, showed that the main cause of graft failure was endothelial dysfunction, with a histopathologically detected ECL in $89.8 \%$ of the failed corneal grafts. Endothelial failure was also documented as the leading cause of graft failure in PKP cases in studies by Alio et al. [18] and Bajracharya et al. [19]. The results of these studies are in accordance with our own, in which endothelial graft failure was documented in 8 cases in total.

In some other studies, the ECL rate was less than that in the present study, where Bertelmann et al. [20] reported an ECL rate of $39.8 \%$ and Bourne [21] reported a rate of $34.2 \%$ at 12 months postoperatively. This may be partially attributed to the larger sample sizes in both of these previous studies (293 and 500 studied grafts, respectively).

The results of the present study are relatively contradictory to those of a study performed by Tan et al., who analyzed the indications, complications, long-term survival rates, and risk factors for PKP graft failure in 901 corneal grafts from Southeast Asia at a single tertiary center [11], which showed that therapeutic and tectonic PKP both carry a worse prognosis in terms of graft survival as compared to optical PKP. Another study, by Anshu et al. [10], also showed better survival for optical PKPs. These contradictory results may be attributed to the plethora of risk factors that were analyzed in these 2 studies (demographic, preoperative, intraoperative, and donor risk factors), many of which were not significantly different between the 2 groups in our study, or were not applicable in our study (such as the concurrent performance of other surgeries). Furthermore, the larger sample size and longer follow-up intervals in these 2 studies may partially explain the different results.

In terms of the good prognosis of therapeutic grafts over time, our study results were similar to those of Xiao et al. [22]. They reported that, for cases of fungal keratitis and herpes simplex keratitis that were treated with therapeutic PKP, only a small, insignificant portion of the transplanted grafts suffered from endothelial decompensation, which gradually declined in rate during the 5 years of follow-up. However, the rate of ECL was not reported in their study. Nevertheless, even though surgeons tend to predict a worse prognosis for therapeutic grafts than for optical grafts, both studies found that therapeutic grafts had a good prognosis.

In our recruited patients of group 2, the addition of antimicrobials for 2-3 weeks following therapeutic PKPs did not have any adverse effects on the postoperative ECL rate, as evidenced by the absence of significant differences between the groups in terms of ECL, except for a small difference after 1 year. Future studies should be conducted on larger cohorts and with longer follow-up intervals, to verify our study results.

The strengths of this study included the strict inclusion and exclusion criteria for the enrolled cohorts. We excluded some patients from the final statistical analysis in the study because they developed factors that could possibly alter the ECL (either graft rejection episodes, raised IOP, or re-infection of the therapeutic grafts). Although excluding those grafts may have omitted some eyes that would show the overall prognosis of optical and therapeutic PKP over time, their exclusion prevented any conflicts in the interpretation of the ECL rate after corneal grafting in both groups. Furthermore, we found no significant difference in the endothelial graft rejection rate between the 2 groups. Nevertheless, our study focused on the ECL rate, and including cases that developed endothelial graft rejection could significantly alter the ECD values. Although endothelial graft rejection was comparable in both groups, studies with larger sample sizes might reveal a significant difference in the rate of graft rejection. Thus, there is a need for future studies with larger samples to verify the rate of graft rejection in optical versus therapeutic PKP.

Although our study results showed a statistically significant, but clinically small increase in the ECL rate in the therapeutic PKP group as compared to the optical PKP group at the 12-months follow-up, further long-term longitudinal studies are needed to determine whether this clinically small change reflects an ongoing process that may progressively affect graft survival or whether it is a static loss rate. Another limitation is the lack of data concerning the morphologic analysis of individual endothelial cells and central corneal thickness for both graft types. Therefore, specular microscopic morphological analysis of individual endothelial cells (coefficient of variation of cell area, and percentage of hexagonal cells), and central corneal thickness in future studies may provide further detail on the condition of the endothelial cells in the 2 graft types.

\section{CONCLUSIONS}

Our study results indicated that therapeutic PKP could be comparable to optical PKP in terms of graft viability, the ECL rate, and the rate of graft rejection along a follow-up interval of 1 year. Hence, therapeutic PKP could be 
a reliable treatment modality for infectious corneal ulcers that are resistant to conventional medical therapies, to avoid devastating complications of recalcitrant infectious keratitis, such as endophthalmitis or panophthalmitis. However, further long-term longitudinal studies are required to confirm this conclusion.

\section{ETHICAL DECLARATIONS}

Ethics approval: The study adhered to the tenets of the Declaration of Helsinki and was approved by the Ethical Committee of Ain Shams University (Ethical code: FWA000017585). All participants were informed about the nature of the study, and signed informed consent prior to participation.

\section{Conflict of interests: None.}

\section{FUNDING}

\section{None.}

\section{ACINOWLED GEMENTS}

This paper has been published previously as a preprint in Research Square Preprint (DOI: 10.21203/ rs.3.rs-294648/v1, March 2021, URL: https://www.researchsquare.com/article/rs-294648/v1).

\section{REFERENCES}

1. Boruchoff SA, Thoft RA, (2005). Keratoplasty: lamellar and penetrating (pp. 645-665). In: Smolin G, Thoft RA, editors. The cornea. Boston, MA: Little, Brown.

2. Duan R, de Vries RD, Osterhaus AD, Remeijer L, Verjans GM. Acyclovir-resistant corneal HSV-1 isolates from patients with herpetic keratitis. J Infect Dis. 2008;198(5):659-63. doi: 10.1086/590668 pmid: 18627246

3. Hernandez-Camarena JC, Graue-Hernandez EO, Ortiz-Casas M, Ramirez-Miranda A, Navas A, Pedro-Aguilar L, et al. Trends in Microbiological and Antibiotic Sensitivity Patterns in Infectious Keratitis: 10-Year Experience in Mexico City. Cornea. 2015;34(7):778-85. doi: 10.1097/ICO.0000000000000428 pmid: 25811724

4. Arnalich-Montiel F, Alio Del Barrio JL, Alio JL. Corneal surgery in keratoconus: which type, which technique, which outcomes? Eye Vis (Lond). 2016;3:2. doi: 10.1186/s40662-016-0033-y pmid: 26783544

5. Bhadange Y, Das S, Kasav MK, Sahu SK, Sharma S. Comparison of culture-negative and culture-positive microbial keratitis: cause of culture negativity, clinical features and final outcome. Br J Ophthalmol. 2015;99(11):1498-502. doi: 10.1136/bjophthalmol-2014-306414 pmid: 25911069

6. Krysik K, Wroblewska-Czajka E, Lyssek-Boron A, Wylegala EA, Dobrowolski D. Total Penetrating Keratoplasty: Indications, Therapeutic Approach, and Long-Term Follow-Up. J Ophthalmol. 2018:9580292. doi: 10.1155/2018/9580292 pmid: 29850220

7. Patel SV, Hodge DO, Bourne WM. Corneal endothelium and postoperative outcomes 15 years after penetrating keratoplasty. Am J Ophthalmol. 2005;139(2):311-9. doi: 10.1016/j.ajo.2004.09.045 pmid: 15733993

8. Claerhout I, Beele H, Kestelyn P. Graft failure: I. Endothelial cell loss. Int Ophthalmol. 2008;28(3):165-73. doi: 10.1007/s10792-0079087-0 pmid: 17508129

9. Ishii N, Yamaguchi T, Yazu H, Satake Y, Yoshida A, Shimazaki J. Factors associated with graft survival and endothelial cell density after Descemet's stripping automated endothelial keratoplasty. Sci Rep. 2016;6:25276. doi: 10.1038/srep25276 pmid: 27121659

10. Anshu A, Li L, Htoon HM, de Benito-Llopis L, Shuang LS, Singh MJ, et al. Long-Term Review of Penetrating Keratoplasty: A 20-Year Review in Asian Eyes. Am J Ophthalmol. 2021;224:254-266. doi: 10.1016/j.ajo.2020.10.014 pmid: 33129808

11. Tan DT, Janardhanan P, Zhou H, Chan YH, Htoon HM, Ang LP, et al. Penetrating keratoplasty in Asian eyes: the Singapore Corneal Transplant Study. Ophthalmology. 2008;115(6):975-982 e1. doi: 10.1016/j.ophtha.2007.08.049 pmid: 18061267

12. User's Manual for the NIDEK SPECULAR MICROSCOPE, CEM-530. TOKYO: NIDEK Corporation (2011). Available at: https:// archive.org/stream/CEM530SmXSM1RDA002A/CEM530_sm_XSM1RDA002A_djvu.txt (Accessed: June 01, 2021).

13. Stamate A-C, Tătaru CP, Zemba M. Emergency penetrating keratoplasty in corneal perforations. Rom J Ophthalmol. 2018;62(4):253-259. doi: $10.22336 /$ rjo.2018.39 pmid: 30891520

14. Obata H, Ishida K, Murao M, Miyata K, Sawa M. Corneal endothelial cell damage in penetrating keratoplasty. Jpn J Ophthalmol. 1991;35(4):411-6. pmid: 1821431

15. Bourne WM, Hodge DO, Nelson LR. Corneal Endothelium Five Years After Transplantation. Am J Ophthalmol. 1994;118(2):185-96. doi: 10.1016/s0002-9394(14)72898-3 pmid: 8053464

16. Nishimura JK, Hodge DO, Bourne WM. Initial endothelial cell density and chronic endothelial cell loss rate in corneal transplants with late endothelial failure. Ophthalmology. 1999;106(10):1962-5. doi: 10.1016/s0161-6420(99)90409-8 pmid: 10519593

17. Eghtedari M, Kamalzadeh M, Yasemi M, Movahedan H, Ashraf MJ. Five Years Pathological Evaluation of Corneal Regrafts: A Study from Southern Iran. J Ophthalmol. 2020:2546923. doi: 10.1155/2020/2546923 pmid: 33489323

18. Alio JL, Montesel A, El Sayyad F, Barraquer RI, Arnalich-Montiel F, Alio Del Barrio JL. Corneal graft failure: an update. Br J Ophthalmol. 2021;105(8):1049-1058. doi: 10.1136/bjophthalmol-2020-316705 pmid: 32788325

19. Bajracharya L, Gurung R. Outcome of therapeutic penetrating keratoplasty in a tertiary eye care center in Nepal. Clin Ophthalmol. 2015;9:2299-304. doi: 10.2147/OPTH.S92176 pmid: 26673279

20. Bertelmann E, Pleyer U, Rieck P. Risk factors for endothelial cell loss post-keratoplasty. Acta Ophthalmol Scand. 2006;84(6):766-70. doi: 10.1111/j.1600-0420.2006.00726.x pmid: 17083535

21. Bourne WM. Cellular changes in transplanted human corneas. Cornea. 2001;20(6):560-9. doi: 10.1097/00003226-200108000-00002 pmid: 11473153

22. Xiao X, Xie L. The influencing factors and characteristics of corneal graft endothelial decompensation after penetrating keratoplasty. Eur J Ophthalmol. 2010;20(1):21-8. doi: 10.1177/112067211002000103 pmid: 19882514 